\title{
Subcellular localization of proteins involved in the assembly of the spore coat of Bacillus subtilis
}

\author{
Adam Driks, ${ }^{1}$ Steven Roels, ${ }^{1}$ Bernard Beall, ${ }^{2}$ Charles P. Moran Jr., ${ }^{2}$ and Richard Losick ${ }^{1}$ \\ ${ }^{1}$ Department of Cellular and Developmental Biology, The Biological Laboratories, Harvard University, Cambridge, \\ Massachusetts 02138 USA; $^{2}$ Department of Microbiology and Immunology, Emory University School of Medicine, Atlanta, \\ Georgia 30322 USA
}

Spores of the bacterium Bacillus subtilis are encased in a two-layered protein shell, which consists of an electron-translucent, lamellar inner coat, and an electron-dense outer coat. The coat protein CotE is both a structural component of the coat and a morphogenetic protein that is required for the assembly of the outer coat. We now show that CotE is located in the outer coat of the mature spore and that at an intermediate stage of sporulation, when the developing spore (the forespore) is present as a free protoplast within the sporangium, CotE is localized in a ring that surrounds the forespore but is separated from it by a small gap. We propose that the ring is the site of assembly of the outer coat and that the gap is the site of formation of the inner coat. Assembly of the ring depends on the sporulation protein SpoIVA, which sits close to or on the surface of the outer membrane that encircles the forespore. We propose that SpoIVA creates a basement layer around the forespore on which coat assembly takes place. The subcellular localization and assembly of CotE and other coat proteins are therefore determined by the capacity of SpoIVA to recognize and adhere to a specific surface within the sporangium, the outer membrane of the forespore.

[Key Words: Bacillus subtilis; coat proteins; morphogenesis; sporulation; subcellular localization]

Received October 12, 1993; revised version accepted November 22, 1993.

A fundamental problem in cellular and developmental biology is the issue of how structures assemble at particular sites within cells (Shapiro 1993). Well-known examples of subcellular localization in microorganisms are the formation of a ring of the Escherichia coli protein FtsZ at the site of cell division (Lutkenhaus 1993), the assembly of a flagellum, and the machinery for chemotaxis at one pole of the Caulobacter crescentus cell (Alley et al. 1993|, and the creation of a complex of cytokinesis proteins at the site of bud formation in Saccharomyces cerevisiae (Chant and Pringle 1991). Another noteworthy example of subcellular localization is the process of spore coat morphogenesis in the bacterium Bacillus subtilis in which the assembly of a structure of complex architecture, the protein shell that will encase the spore, occurs at a particular site within the developing sporangium (Piggot and Coote 1976; Losick et al. 1986).

Spore formation involves the partitioning of the developing cell (or sporangium) into unequal-sized cellular compartments called the mother cell and the forespore by the formation of an asymmetrically positioned septum (Fig. 1B). After the septation stage of sporulation, the edges of the septum migrate toward the forespore pole of the sporangium, engulfing the forespore and eventually pinching it off as a free protoplast in the mother-cell cytoplasm (Fig. 1C,D). When engulfment is complete, a thick layer of cell wall material called the cortex is produced in the space between the double membrane layers that surround the forespore (Fig. 1E). Finally, a complex protein shell called the coat is assembled around the mother-cell membrane that surrounds the forespore (Fig. 1F). Electron microscopy reveals two major substructures in the coat: a lightly staining inner coat that is lamellar in appearance and a darkly staining outer coat (Fig. 2A; Aronson and Fitz-James 1976). The coat protects the spore after it is released into the environment, and it is also involved in the response to conditions that trigger germination. The completion of the coat is the final stage in sporulation, and it is followed by lysis of the mother cell and release of the mature spore.

Sporulation is driven by a precisely controlled program of gene expression that bifurcates, after the sporulation septum is laid down, into two separate but coupled programs, one in each of the newly formed compartments (Losick and Stragier 1992). These distinct pathways of gene expression allow the spore to be constructed in a stepwise fashion, from both the outside in and from the inside out, as different spore components are made available only at specific times, and in the correct compartment. This control is achieved, in large part, by the sequential appearance of sporulation-specific transcription 


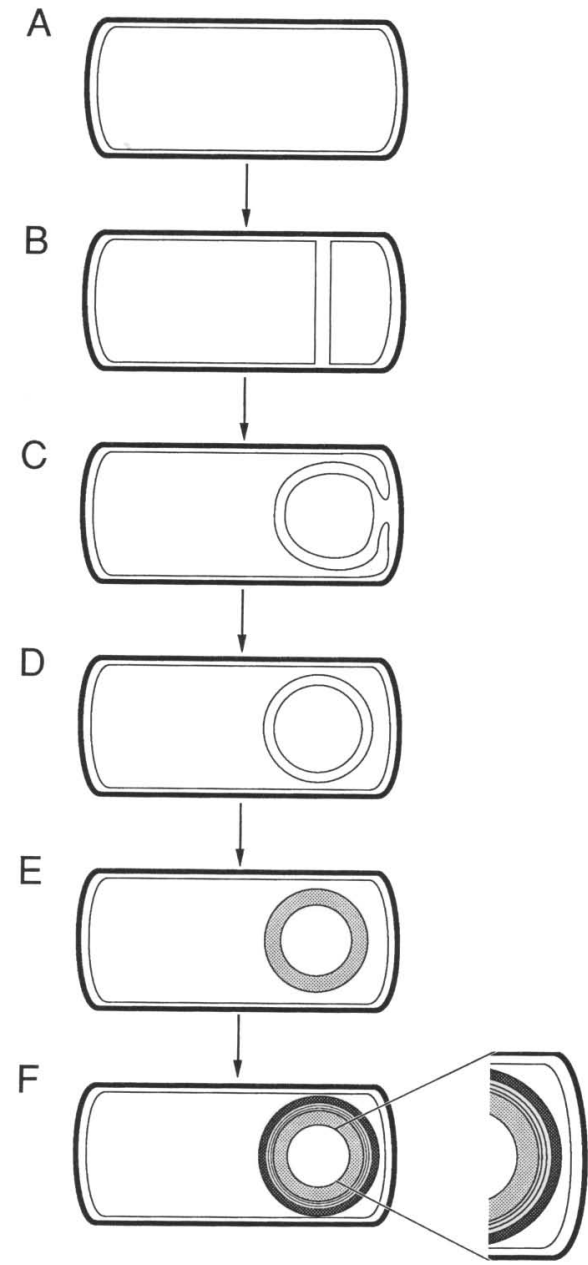

Figure 1. Stages of sporulation. (A) A sporangium prior to the formation of a septum. The cell wall is indicated by a thick line, and the inner membrane by a thin line. $(B)$ A sporangium at the stage of septation. $(C)$ A sporangium in which the first step in engulfment, the migration of the septum toward the forespore pole, has occurred. $(D)$ A sporangium in which engulfment is complete and the forespore is pinched off as a free protoplast in the mother cell. $(E)$ A sporangium at the stage of cortex formation, indicated as the shaded region between the forespore membranes. $(F)$ The sporangium at the stage of coat formation. The coat is composed of two layers, a lamellar inner coat (represented as thin lines surrounding the forespore) and a darkly staining outer coat (represented as a dark area surrounding the inner coat). A blowup of the right-hand edge of the sporangium is provided so that the details of the coat can be seen.

factors. Here, we are concerned with the program of gene expression in the mother cell, the compartment in which assembly of the coat takes place. The mother-cell line of gene expression commences with the action of transcription factor $\sigma^{\mathrm{E}}$ (Zheng and Losick 1990; Driks and Losick 1991), which directs the expression of genes that encode proteins involved in both cortex and coat assembly, in particular the sporulation gene spoIVA (Roels et al. 1992; Stevens et al. 1992) and the coat protein gene $\cot E$ (Zheng and Losick 1990). It is also respon- sible for the expression of spoIIID (Kunkel et al. 1989), which encodes a DNA-binding protein that directs the appearance of a third transcription factor called $\sigma^{\mathrm{K}}$ (Kroos et al. 1989). The $\sigma^{\mathrm{K}}$ factor, in turn, is responsible for the expression of coat protein genes $\cot A, \cot D, \cot F$ (Cutting et al. 1991b), and $\cot G$ (E. Ricca, S. Cutting, and R. Losick, unpubl.) and the regulatory gene gerE (Cutting et al. 1989|. Finally, the product of gerE is a DNA-binding protein that switches on the transcription of the last known temporal class of mother-cell-specific gene expression (Zheng and Losick 1990; Zheng et al. 1992). Among the genes that are activated by GerE are the coat protein genes $\cot B$ and $\cot C$.

The CotE protein plays a key role in the assembly of the coat (Zheng et al. 1988). It is not only a structural component of the coat but is also required for the formation of the outer layer of the coat; a $\cot E$ null mutant produces spores that lack not only CotE but also the other protein components of the outer coat. We now report that CotE assembles into a ring around the nascent forespore at relatively early stages of sporulation, prior to the time of synthesis of all other known coat proteins. This ring is separated from the mother-cell membrane that surrounds the forespore by a small gap that will become the inner coat. Assembly of the CotE ring depends on spoIVA, mutations in which were known to cause the coat to misassemble as swirls in the mothercell cytoplasm rather than around the forespore (Piggot and Coote 1976; Roels et al. 1992). We demonstrate further that SpoIVA, itself, is present around the forespore, lying close to or on its outer surface. Thus, SpoIVA appears to be responsible for guiding the subcellular localization of coat assembly by providing a basement layer on which coat assembly can occur. Finally, we show that

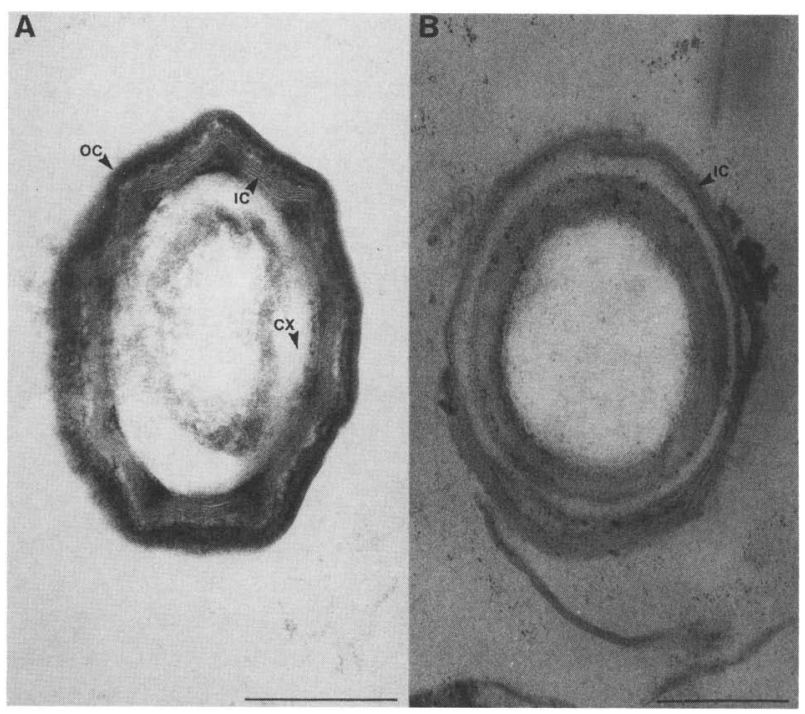

Figure 2. Electron microscopy of wild-type and mutant spores. (A) A wild-type spore. Inner coat (IC), outer coat (OC), and cortex $(C X)$ are labeled. $(B)$ A spore from a $\cot E$ mutant in which the outer coat is missing, leaving only the inner coat (IC). Bars, $250 \mathrm{~nm}$. 
another morphogenetic protein SpoVID is required for the continued localization of $\operatorname{CotE}$ to the region around the forespore.

\section{Results}

Immunoelectron microscopic localization of CotE in spores

The coat of wild-type B. subtilis spores consists of two principal layers: a lightly staining lamellar inner coat and a more electron-dense outer coat (Fig. 2A). Proper formation of the coat depends on the CotE protein. Spores from a $\cot E$ mutant lack the outer coat (Zheng et al. 1988) (Fig. 2B) and frequently display an aberrant inner coat that appears to be disconnected from the outer surface of the forespore. Because of the critical role of CotE in coat assembly, it was of interest to determine the location of this morphogenetic protein within the coat.

To do this, we carried out immunoelectron microscopy experiments using a monoclonal antibody to detect a modified form of CotE bearing the HAl epitope (Field et al. 1988). Spores were fixed and embedded, and thin sections were treated with the anti-HAl antibody. After application of gold-tagged secondary antibodies and silver enhancement of the gold grains, the sections were stained with heavy metal and examined in the electron microscope. To create the epitope-tagged form of CotE, the DNA sequence specifying the HAl epitope of hemagglutinin was inserted in-frame at the $3^{\prime}$ end of a cloned copy of the $\cot E$ gene, a position at which the insertion was unlikely to severely impair CotE function as inferred from an earlier investigation showing that the carboxyl terminus of CotE is dispensable (Zheng et al. 1988). The modified $\cot E$ gene was then used to replace the wild-type copy of the gene in the chromosome. Western blotting using a monoclonal antibody against the epitope revealed a protein the same size as CotE in proteins extracted from sporulating cells bearing the modified gene, but not in protein from cells containing the wild-type $\cot E$ gene (data not shown).

As confirmation that the epitope-tagged CotE was functional, electron microscopy showed that spores from a strain bearing the modified $\cot E$ gene exhibited relatively normal-looking inner and outer coats. These spores were not, however, entirely normal in that more frequently than in the wild type the outer coat failed to fully encircle the spore, resulting in bare regions where only inner coat appeared (Fig. 3A,B).

Immunoelectron microscopy of free spores and of latestage sporangia bearing fully mature spores showed that CotE was predominantly located in the coat, the gold grains often being located at the interface between the inner and outer coat layers. Interestingly, gold grains did not significantly decorate those regions of the coat that were missing the outer coat, a finding that suggested that CotE is an outer coat component (Fig. 3A,B). As a further indication that CotE is in the outer coat, immunoelectron microscopy showed that CotE was present in the

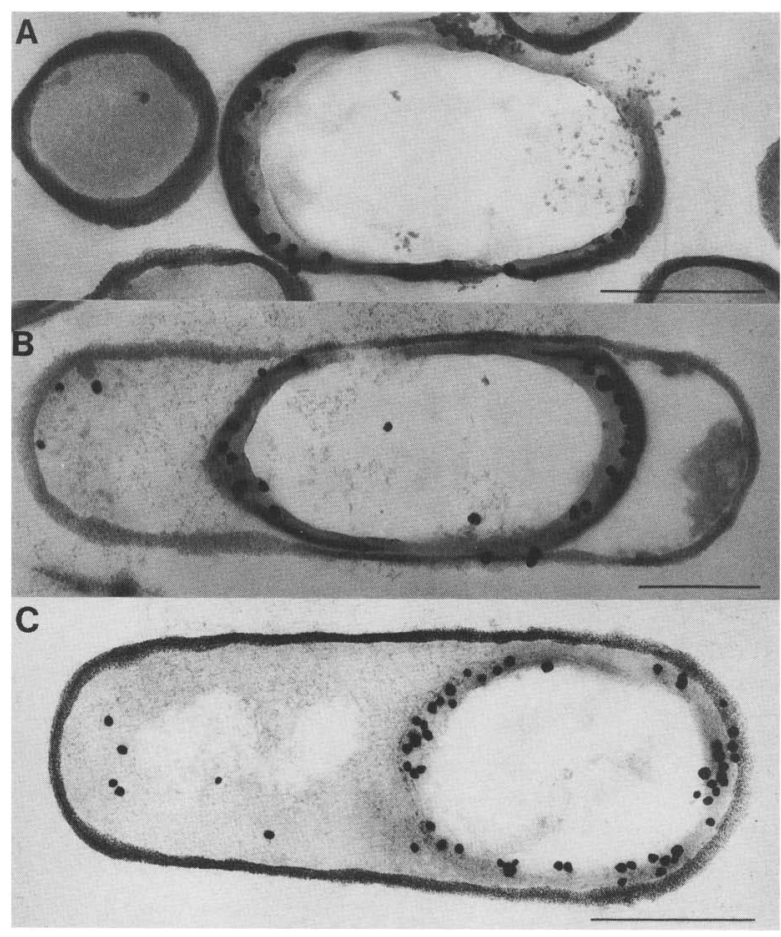

Figure 3. Immunoelectron microscopic localization of CotE. (A) A free spore; $(B)$ a late-stage sporangium. Both were obtained from a wild-type strain. (C) A sporangium of a gerE mutant strain, which is therefore missing the inner coat. Immunoelectron microscopy was carried out using antiepitope antibody and cells producing epitope-tagged CotE, as described in Materials and methods. Bars, $500 \mathrm{~nm}$.

coat of spores of a gerE mutant, which is known to be specifically lacking the inner coat (as illustrated by the spore contained within the mutant sporangium in Fig. 3C) (Moir 1981).

Note that the immunoelectron microscopy images show less morphological detail than electron microscopy images from experiments designed to reveal spore ultrastructure (e.g., cf. Fig. 2A with Fig. 3A). We used two methods to prepare cells for electron microscopy. For morphological analysis (e.g., Fig. 2), a lengthy fixation and dehydration protocol was used (Margolis et al. 1993), giving good structural preservation. For immunoelectron microscopy, the cells were subjected to gentle fixation and dehydration to maximize the preservation of antigenicity.

\section{Subcellular location of CotE early in sporulation}

CotE is produced at a middle stage of sporulation, prior to the time at which a morphologically recognizable coat can be detected. To study the subcellular location of CotE at this intermediate time, we carried out immunoelectron microscopy using mutants blocked at the engulfment stage of sporulation (Fig. 1D). Such mutants carry out engulfment of the forespore but are unable to produce a cortex or a coat and are blocked in the expres- 
sion of all known coat protein genes except $\cot E$. Immunoelectron microscopy indicated that CotE in three such mutants [spoIIID, spoIIIG (see Fig. 4) and spoIIIA (data not shown)] was primarily located in the vicinity of the forespore (Fig. 4). Interestingly, the gold grains often appeared to be arranged in a ring separated from the outer edge of the forespore by a distance of $\sim 75 \mathrm{~nm}$. A similar result was obtained when $s p o^{+}$cells at this same stage of development were examined (data not shown). An interesting feature of the images in Figure 4 is the apparent tendency of the gold grains to cluster at the poles of the forespore. This is reminiscent of the time course of the appearance of the coat later in development, when inner and outer coat initially appear at the forespore poles and then proceed to encircle the spore (A. Driks and R. Losick, unpubl.).

We then wished to determine the location of CotE at septation, the stage (II) prior to engulfment when the septum has not yet migrated around the forespore (Fig. 1B). Immunoelectron microscopy was carried out using three stage II mutants, each one representing a different substage of septal morphogenesis. When cells enter stage II, the septum is straight or slightly curved and two membrane layers separated by a gap can be discerned; this gap then disappears. Finally, the process of engulfment begins as the septum begins to migrate toward the forespore pole. The double mutant spoIIB spoVG (Mar-

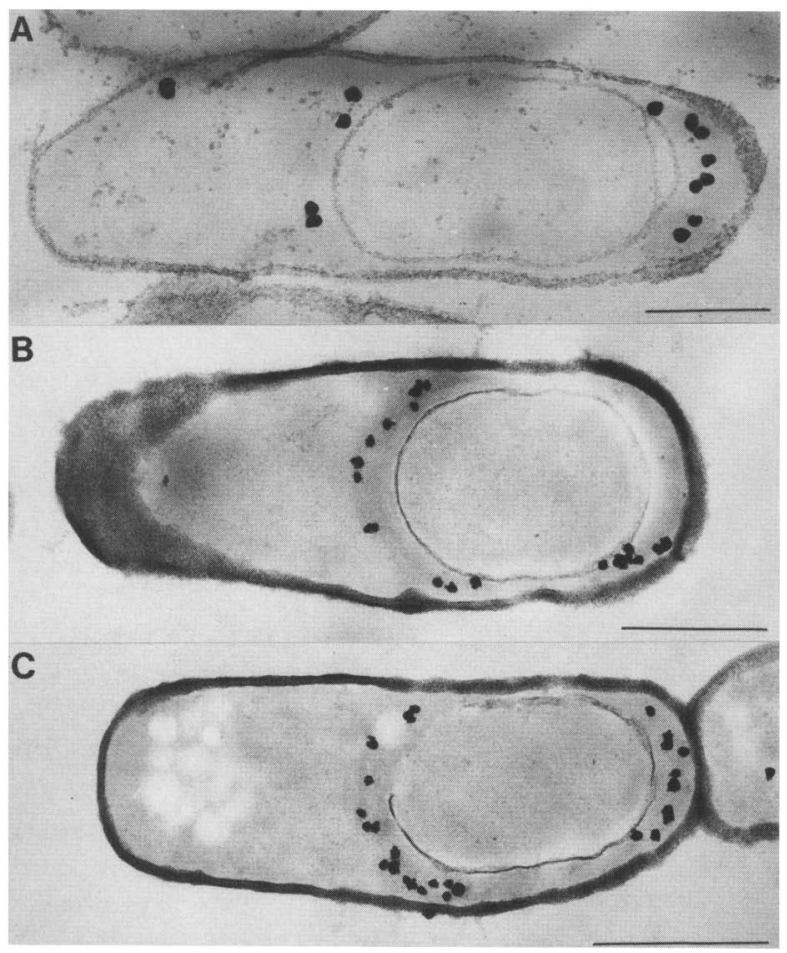

Figure 4. Immunoelectron microscopic localization of CotE at the stage of engulfment. (A) A sporangium from a spoIIID mutant; $(B, C)$ sporangia from a spoIIIG mutant. Immunoelectron microscopy was carried out as described in the legend to Fig. 3 and in Materials and methods. Bars, $500 \mathrm{~nm}$.

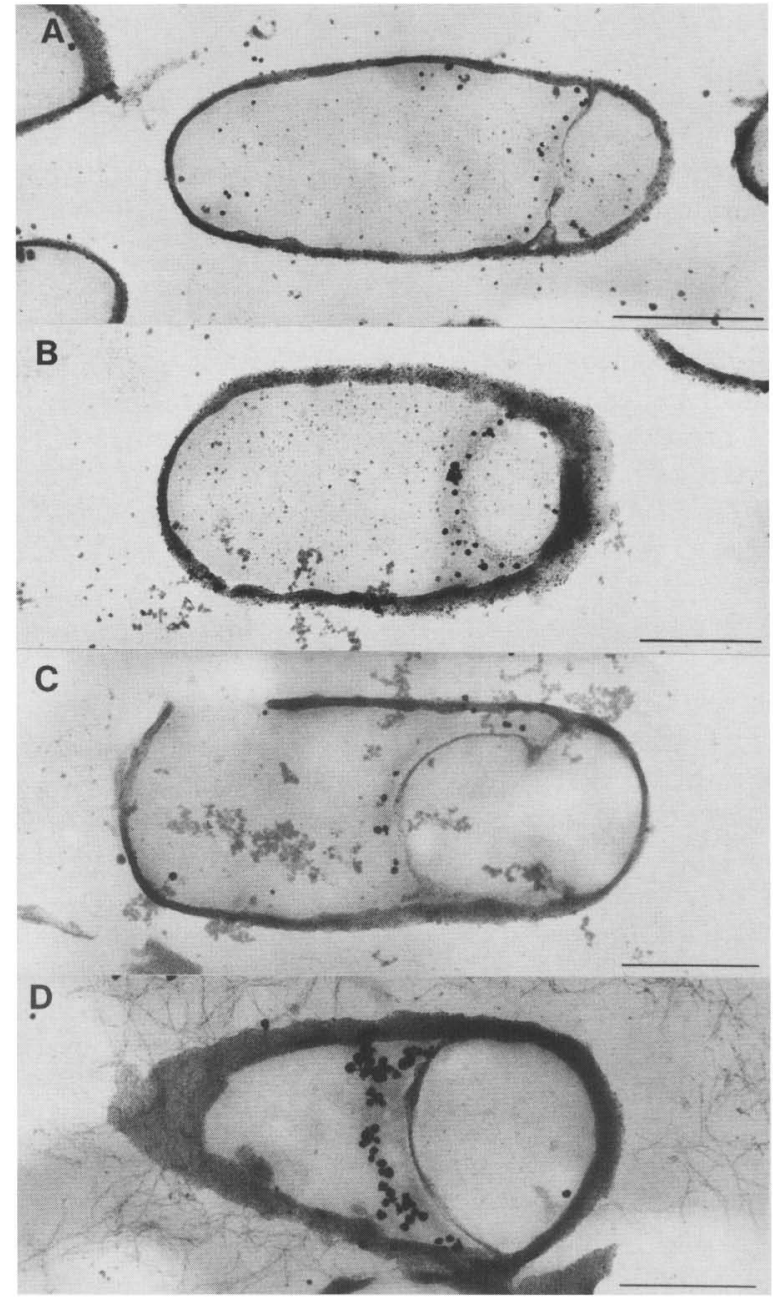

Figure 5. Immunoelectron microscopic localization of CotE at the stage of septum formation. $(A, B)$ Sporangia from a $s p o I I B$ spoVG mutant. (C) A sporangium from a spolID mutant; $(D) \mathrm{A}$ sporangium from a spoIIAC mutant. Note that the light fixation and embedment conditions used for immunoelectron microscopy did not permit visualization of the detailed morphology of these mutants, such as the gap in the septa of sporangia from a spoIIB spoVG mutant. Although the lead stain gave the cell envelope a mottled appearance reminiscent of gold grains, in original micrographs this punctate staining is easily distinguished from actual decoration. The variation in gold grain size was a result of the silver enhancement protocol (see Materials and methods). Immunoelectron microscopy was carried out as described in the legend to Fig. 3 and in Materials and methods. Bars, $500 \mathrm{~nm}$.

golis et al. 1993) is blocked at the earliest substage of septal development, whereas strains bearing a mutation in spoIID (Coote 1972) or the mutation spoIIAC561 (Illing and Errington 1991) undergo septal thinning but are unable to complete engulfment. In each of these mutants, gold grains were found to be clustered along the septum but often separated from it by a short distance (Fig. 5). Thus, subcellular localization of CotE is an early event in sporulation requiring neither thinning of the 
sporulation septum nor migration of the septum around the forespore. Because CotE was not located flush against the septal or engulfment membranes, but was separated from it by a small distance, we infer that some structure must exist that holds CotE away from the surface of the membrane. We often observed a lightly staining arc (or circle) in this region (e.g., Figs. 4, 5, and 9) that could represent such a structure. Interestingly, we do not observe this feature when cells are prepared with the standard fixation method used for conventional morphological analysis. As far as we are aware, this structure has not been explicitly noted previously [although we believe it can be observed in Fig. 8F in Hobot et al. (1985), who used a mild fixation and dehydration protocol similar to ours].

We wished to determine what gene products might be involved in sequestering CotE to the region around the forespore. An attractive candidate is the product of the sporulation gene spoIVA, which like $\cot E$ is induced early in the mother-cell line of gene expression under the control of $\sigma^{\mathrm{E}}$. Mutants of this gene exhibit an unusual phenotype: A normal-looking coat is formed (having the characteristic inner and outer layers), but instead of attaching to the forespore, the coat misassembles as detached swirls in the mother-cell cytoplasm (Piggot and Coote 1976; Roels et al. 1992). To determine whether the subcellular localization of CotE was dependent on the product of spoIVA, we introduced a spoIVA mutation into strains bearing mutations in spoIIID, spoIID, or spoIIAC and carried out immunoelectron microscopy. In these experiments we found that the gold grains were not clustered near the forespore surface but were instead present in the mother-cell cytoplasm, where they tended to be grouped into clumps (Fig. 6). As expected, in a strain bearing only the spoIVA mutation, gold grains appeared in the swirls of coat in the mother-cell cytoplasm (data not shown).

\section{SpoIVA is located at the forespore surface}

The results described above raised the possibility that SpoIVA, itself, is located at the surface of the forespore from which location it directs CotE and other coat proteins to assemble around the forespore. To investigate this hypothesis, we raised antibodies against SpoIVA. The anti-SpoIVA antibodies were used to localize the protein by means of immunoelectron microscopic analysis of mutant cells blocked either at the stage of engulfment or at the stage of septum formation. Although some gold grains appeared throughout the mother-cell cytoplasm, a high proportion were clustered at the septal membrane (Fig. 7B,C) or around the forespore (Fig. 7A,D). In contrast to CotE, which was localized in a ring separated from the forespore by a small distance, SpoIVA appeared to be located close to or right at the surface of the membrane surrounding the forespore (e.g., Fig. 7D). Similar results were obtained when wild-type cells at the stage of engulfment or at the stage of septum formation were examined (data not shown).

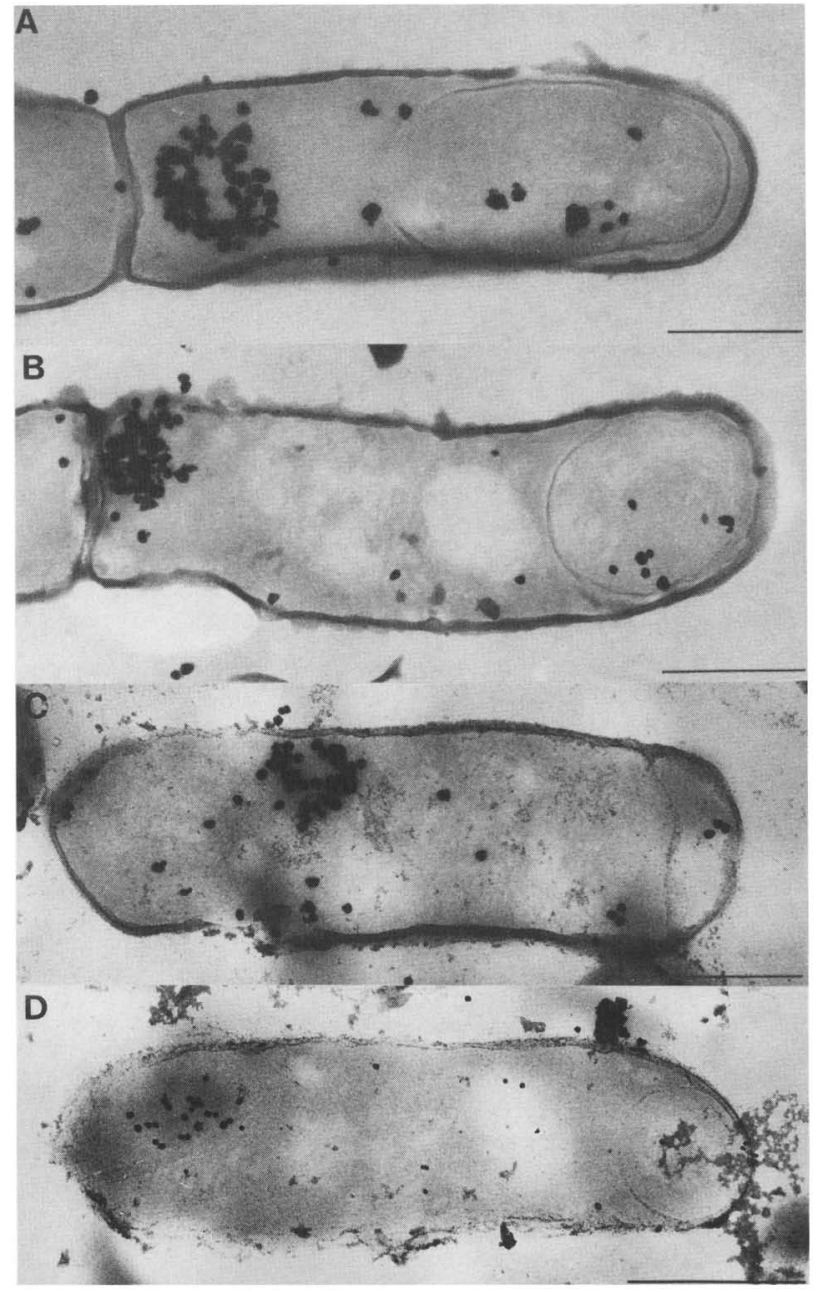

Figure 6. Immunoelectron microscopic localization of CotE in the presence of a spoIVA mutation. $(A, B)$ Sporangia from a spoIIID spolVA mutant; the sporangium in $C$ is from a spoIID spoIVA mutant; the sporangium in $D$ is from a spoIIAC spoIVA mutant. Immunoelectron microscopy was carried out as described in the legend to Fig. 3 and in Materials and methods. Bars, $500 \mathrm{~nm}$.

\section{Effect of a spoVID mutation on the localization of CotE and SpoIVA}

A second gene whose product is likely to be involved in localizing CotE to the forespore region is spoVID (Beall et al. 1993). A mutation in spoVID results in the formation of the coat as swirls in the mother-cell cytoplasm instead of around the forespore, similarly to the effect of the spoIVA mutation. To determine whether a mutation in spoVID would prevent CotE localization at the stage of engulfment, we carried out immunoelectron microscopy using a strain bearing mutations in spoIIID and spoVID. Unexpectedly, and in contrast to the effect of a mutation in spoIVA, a mutation in spoVID did not prevent CotE from binding to the region around the forespore (Fig. 8A). However, when the effect of the spoVID mutation was examined in sporangia from strains that 


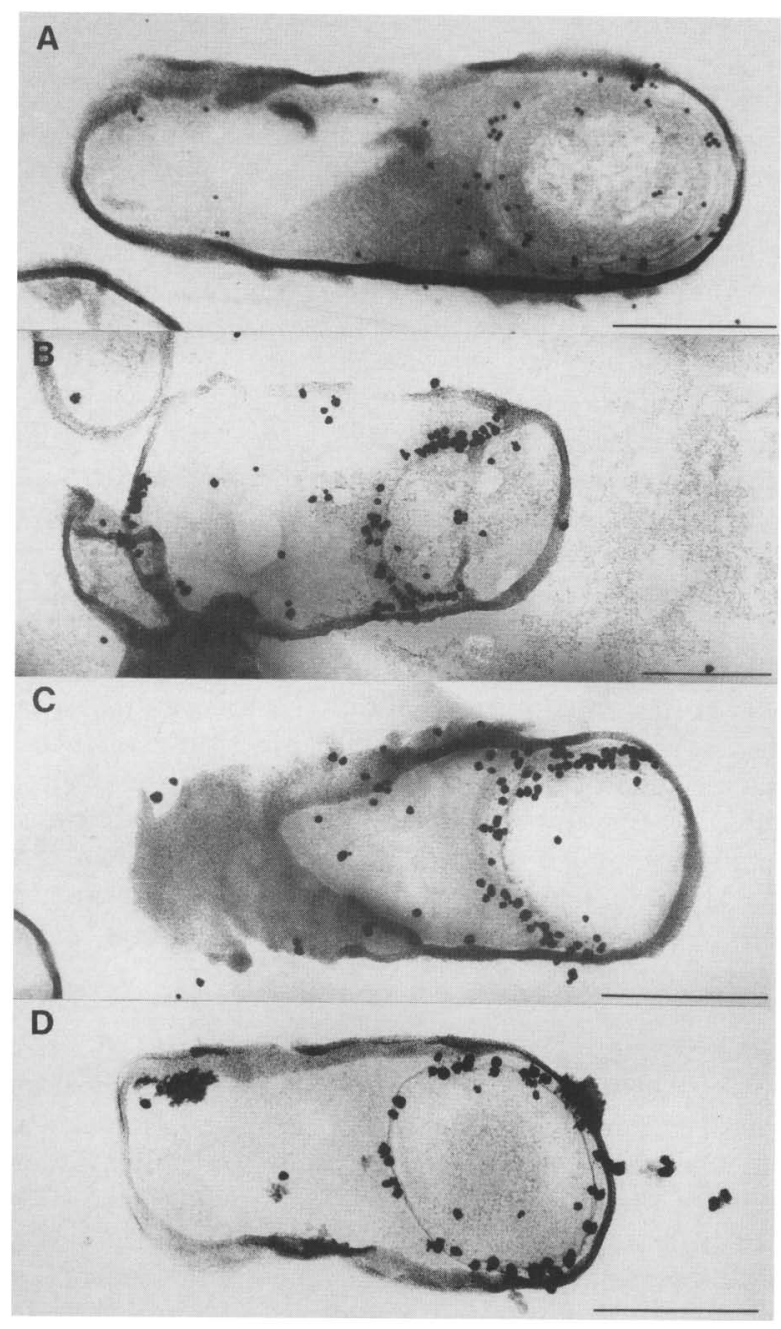

Figure 7. Immunoelectron microscopic localization of SpoIVA. (A) A sporangium from a spoIIID mutant; $(B)$ A sporangium from a spoIID mutant; $(C)$ A sporangium from a spoIIAC mutant; and $(D)$ A sporangium from a spoVID mutant. Immunoelectron microscopy was carried out using polyclonal antibodies raised against purified SpoIVA as described in Materials and methods. Bars, $500 \mathrm{~nm}$.

did not contain a spoIIID mutation and therefore could proceed to a late stage of development, gold grains were associated with coat material in the cytoplasm and not with the forespore (Fig. 8B).

This implies that in a spoVID mutant, CotE localizes at an early time to the region around the forespore, but at some later time (at or just before the appearance of the coat) CotE is no longer able to remain attached. We interpret this to indicate that SpoIVA is required for the initial localization of CotE but that at later times the continued attachment of the assembling coat to the outer surface of the forespore requires the action of SpoVID. In this view, the primary event in recognition of the outer surface of the forespore is the localization of SpoIVA, which we predict should not be dependent on SpoVID. In confirmation of this expectation, we find that the presence of the spoVID mutation did not prevent proper localization of SpoIVA (i.e., at the outer membrane of the forepsore) at the time of engulfment (Fig. 7D) or after the appearance of the coat (data not shown).

\section{Subcellular localization of SpoVID}

The discovery that SpoVID is required for maintaining the association of CotE and the other coat proteins with the forespore raised the question of the subcellular location of SpoVID itself. To investigate this, we created an epitope-tagged version of the protein. The DNA sequence encoding the HAl epitope (Field et al. 1988) was inserted in-frame into spoVID, and the altered spoVID gene substituted for the wild-type copy in the chromosome. Strains bearing the modified spoVID gene produced normal spores, as judged by light microscopy, lysozyme resistance, and germination efficiency (data not shown). When sporangia that synthesized the epitope-tagged SpoVID were analyzed by immunoelectron microscopy, the majority of cells showed no decoration at all (unlike our results with CotE and SpoIVA, in which the majority of the cells showed decoration; see Materials and methods|. However, gold grains were located at the forespore membrane in a very few cells, all of which were at the engulfment stage (Fig. 9). We do not know why the frequency of decorated cells was low, but we take this result to be a tentative indication that SpoVID is present at the forespore surface.

A simple expectation that follows from our results is that the localization of SpoVID is also determined by SpoIVA. However, because we could observe so few ex-

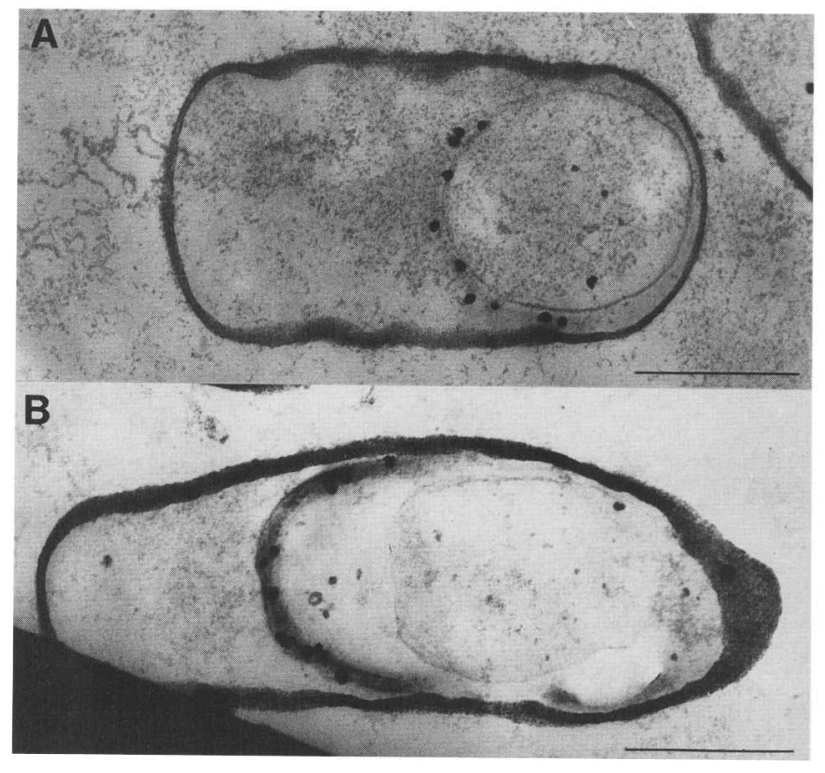

Figure 8. Immunoelectron microscopic localization of CotE in a spoVID mutant. The sporangium in $A$ is from a spoIIID spoVID mutant. The sporangium in $B$ is from a spoVID mutant. Immunoelectron microscopy was carried out as described in the legend to Fig. 3 and in Materials and methods. Bars, $500 \mathrm{~nm}$. 


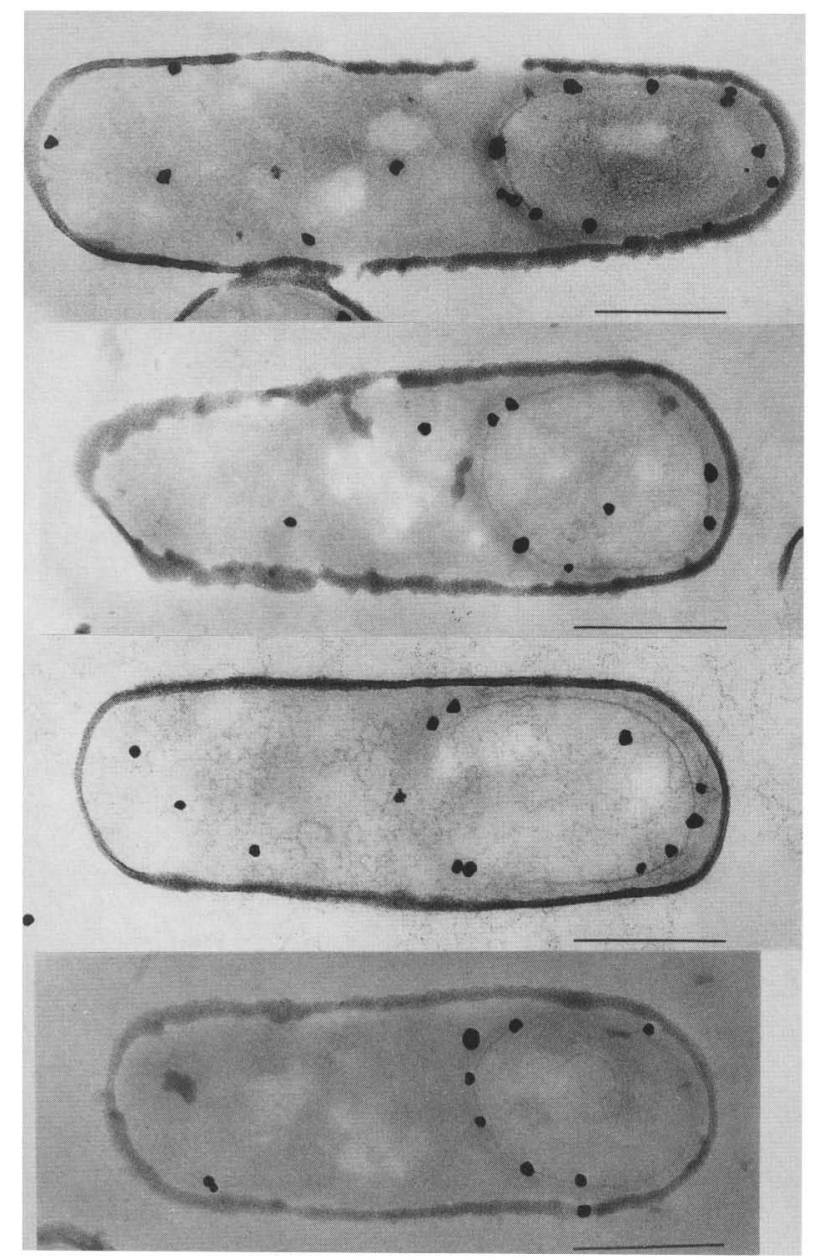

Figure 9. Immunoelectron microscopic localization of SpoVID. Immunoelectron microscopy was carried out using an antiepitope antibody and a $\mathrm{poO}^{+}$strain that produced epitope-tagged SpoVID, as described in Materials and methods. Bars, $500 \mathrm{~nm}$.

amples of SpoVID immunolocalization, we were unable to use immunoelectron microscopy to determine whether the presence of a spoIVA mutation would prevent SpoVID localization.

\section{Discussion}

The expression of genes encoding structural components of the spore coat is governed by a hierarchical regulatory cascade in the mother-cell compartment of the sporangium (Zheng and Losick 1990; Driks and Losick 1991). The coat protein gene $\cot E$ is switched on in the earliest phase of the mother-cell line of gene expression under the control of the transcription factor $\sigma^{\mathrm{E}}$, whereas all other coat protein genes so far identified are induced later in the cascade under the control of the transcription factors $\sigma^{\mathrm{K}}$ and GerE. The use of immunoelectron microscopy and mutants blocked in the appearance of $\sigma^{\mathrm{K}}$ and GerE has enabled us to detect a series of preliminary events in the assembly of the coat that precede any previously detected manifestation of coat morphogenesis. The most striking of these events is the incorporation of CotE into a ring-like structure that surrounds the forespore at the engulfment stage of development (or an arclike structure that abuts the septum in the pre-engulfment sporangium) (Fig. 10). Because this ring is separated from the outer surface of the forespore by a small gap and because CotE is located in and is required for the assembly of the outer coat, we infer that the ring of CotE protein is the site at which the outer coat proteins will assemble at later morphogenetic stages. We speculate that the gap between CotE and the membrane surrounding the forespore is the site at which the inner coat will later assemble. We do not believe that this gap is empty but, rather, that it contains a scaffold that holds the shell of CotE in place and into which inner coat protein will later infiltrate to create the lamellar inner structure of the coat. Under conditions of mild fixation and dehydration, a faintly visible (partially electron-translucent) structure can be detected in the position of the gap that could correspond to such a scaffold. Because all known protein components of the coat except CotE are produced late in the mother-cell line of gene expression, the scaffold cannot be composed of the product of a known coat protein gene.

How are the CotE ring and this putative scaffold guided to the outer surface of the forespore? We have shown that these morphogenetic events are determined, at least in part, by the action of the sporulation protein SpoIVA. It has been recognized since the classic experiments of Piggot and Coote (1976) and confirmed in recent ultrastructural studies (Roels et al. 1992) that SpoIVA plays a special role in coat morphogenesis: Unlike many other sporulation mutations, spoIVA muta-

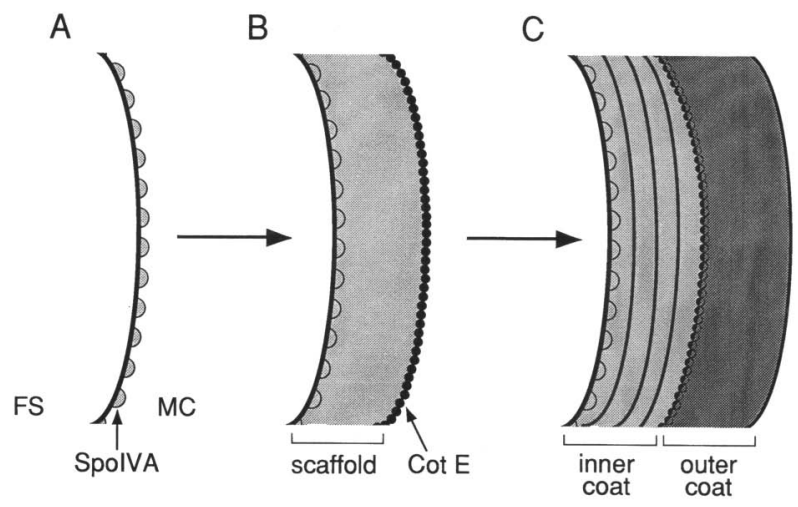

Figure 10. A model for assembly of the coat. We hypothesize that the coat is assembled in three stages. In the first stage $|A|$ SpoIVA (represented by semicircles) binds to the outer membrane (mother-cell derived) surrounding the forespore (indicated by a dark line). The forespore (FS) and mother-cell (MC) sides of the membrane are indicated. In the second stage $(B)$, the scaffold (shown as a light gray region) and CotE (indicated by black dots) appear around the forespore. In the final stage $(C)$, the outer coat (indicated by the dark gray area) and the inner coat (represented by the striations within the scaffold) are formed. 
tions do not prevent the formation of the coat but, rather, impair the mechanisms that guide the assembly process to the outer surface of the forespore, such that the coat [which otherwise looks normal in its ultrastructural detail (Roels et al. 1992)] misassembles as swirls within the mother-cell cytoplasm. It was therefore anticipated that SpoIVA, itself, might be located on the membrane around the forespore where it could serve as a basement structure on which the coat would assemble. In support of this hypothesis, we have shown that the assembly of CotE into a ring around the forespore is dependent on SpoIVA and that SpoIVA, itself, is localized in close apposition to the membrane that encases the forespore. (We speculate that the assembly of the CotE ring and the inner coat depends on a scaffold. If this is so, then, although SpoIVA is required for attachment of the scaffold to the forespore, it cannot be an essential component of the scaffold itself, because spoIVA mutants produce a coat with normal-looking inner and outer coat layers.)

One of the most intriguing unanswered questions raised by our study is how the localization of SpoIVA to the outer forespore membrane is achieved. We note that the mother-cell membrane that engulfs the forespore is identical in orientation to the cytoplasmic membrane of the mother cell from which it was derived (Fig. 1B-D). Furthermore, localization of SpoIVA at this membrane can be observed as early as the stage of septation when SpoIVA can be seen to be concentrated at the mother-cell surface of the polar septum, the membrane that will later migrate around and eventually engulf the forespore. We hypothesize that some special feature of the septal membrane sequesters SpoIVA at this surface as opposed to the remaining surface of the mother-cell cytoplasmic membrane. We do not know what this feature is, but we note that the cytoplasmic membrane is potentially in communication with the environment outside the cell whereas the septal membrane is juxtaposed against the membrane that contains the forespore cytoplasm.

Another intriguing aspect of SpolvA is its dual functionality. In addition to guiding proper localization of the coat, SpoIVA is also required for the formation of the cortex, the layer of cell-wall-like material that is produced in the space between the inner (forespore-derived) and outer (mother-cell-derived) membranes surrounding the forespore following engulfment. Our discovery that SpolVA is located around and possibly in contact with the outer membrane suggests that it could play a direct role in controlling or catalyzing the synthesis of the cortex. Coat and cortex formation can occur independently of one another; a spoVE mutant possesses an aberrant cortex (Piggot and Coote 1976) but does have an apparently normal coat, and, similarly, a $d a c B$ mutant has a defective cortex but is resistant to chloroform and lysozyme (Buchanan and Gustafason 1992), an indication of a functional coat. A strain bearing mutations in both gerE and $\cot E$, on the other hand, produces a cortex but not a coat (A. Driks and R. Losick, unpubl.). Even though coat and cortex formation can occur independently of one another, the role played by SpoIVA in both could indicate that it functions to coordinate the two most distinctive morphological events of spore formation.

As in the case of a spoIVA mutant, sporangia from a spoVID mutant synthesize a coat that does not attach to the forespore but, instead, is present as swirls in the mother-cell cytoplasm. However, in contrast to SpoIVA, the role of SpoVID can only be discerned at a late time in sporulation. In a sporangium from a spoVID mutant at the stage of engulfment, CotE appears in a ring around the forespore, as it would in a wild-type sporangium. When the later stages of development-characterized by the appearance of the cortex and coat-are reached, CotE and other coat material evidently detach from the forespore. Therefore, SpoVID is required for maintaining the proper localization of CotE during the period when the cortex and the coat are being formed. Perhaps the synthesis of either or both structures alters SpoIVA or another scaffold component such that SpoVID is then needed to hold the coat to the forespore.

The direct involvement of SpoIVA and SpoVID in coat assembly raises the question of whether these proteins are bona fide coat proteins; that is, are they components of the completed coat of the mature spore? To address the issue of whether SpoIVA is a coat protein, spores were examined with immunoelectron microscopy using anti-SpoIVA antibodies. The level of gold grains observed was not significantly greater than background, indicating that SpolVA is not a major component of the spore. Likewise for the case of SpoVID, Western blotting experiments indicated that SpoVID is not present in detectable amounts in spores (data not shown). SpoIVA and SpoVID appear not to be coat proteins but, rather, morphogenetic proteins that are only transiently associated with the assembling coat.

Although the regulation of coat protein gene expression is well understood, the mechanism of coat assembly has remained obscure. A simple model for the construction of the coat would have the inner coat built first, followed by the assembly of the outer coat. In such a model, one might expect that inner coat protein genes would be transcribed early, to be followed by the expression of the outer coat protein genes later. However, previous studies have shown that the expression of inner coat protein genes does not necessarily precede that of outer coat protein genes, nor are all the genes encoding the proteins comprising any one layer of the coat transcribed within a single temporal class of gene expression. For example, the first known coat protein gene to be expressed, $\cot E$, encodes a component of the outer coat, and the formation of the inner coat as well as the outer coat depends on the expression of genes in the latest known temporal class, under the control of the transcription factor GerE (Zheng et al. 1988; Zheng and Losick $1990)$. Furthermore, $\cot D$, encoding an inner coat protein, and $\cot A$, encoding an outer coat protein, are expressed simultaneously, at an intermediate time, under the control of the transcription factor $\sigma^{\mathrm{K}}$ (Zheng et al. 1988; Zheng and Losick 1990). The model of coat morphogenesis to emerge from the results reported here sug- 
gests a resolution to this paradox. Rather than the coat being assembled in a linear fashion, layer by layer, a nascent inner and outer coat structure is built first, in the form of a scaffold surrounded by CotE (Fig. 10). As sporulation continues and other coat proteins are synthesized, they are incorporated into this structure, giving rise to the mature inner and outer layers of the coat.

In summary, we propose that the earliest event in the assembly of the coat is the formation of a basement layer of the SpoIVA protein around the outside surface of the forespore (Fig. 10). This basement layer then guides the attachment of a scaffold that surrounds the forespore. This scaffold, in turn, becomes encased in a ring of CotE. Inner coat proteins then infiltrate into the scaffold to create the lamellar layer of the coat, whereas outer coat protein binds to the CotE ring to create the electrondense outer layer. An additional morphogenetic protein, SpoVID, that may attach to the outer surface of the forespore, is required to hold the assembling coat in place at late stages of morphogenesis.

\section{Materials and methods}

\section{Strains}

All strains are congenic derivatives of the wild-type strain PY79 (Youngman et al. 1984). The following sporulation mutant strains

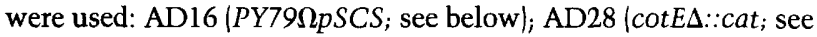
below); PM84 (spoIIAC561::spoIIACRerm; Margolis et al. 1991); PM740 |spoIIBA::erm spoVG::Tn917RHU265; Margolis et al. 1993); RL64 (spoIID::Tn917SHU298; Sandman et al. 1987); RL68 (spoIIIA::Tn917RHU25; Sandman et al. 1987); RL244 (spoIIIDAermG; Cutting et al. 1991a) RL325 (gerE36; Cutting et al. 1989); SC500 (spoIIIGA1; Kunkel et al. 1988); SR263 (spoIVAA::neo1; see below).

To build AD28, DNA from a strain bearing $\cot E \Delta:$ :cat (Zheng et al. 1988) was used to transform PY79 to chloramphenicol resistance.

To construct a null mutation in spoIVA, we first built a plasmid in which the sequence of the spoIVA open reading frame was replaced with the sequence of a neomycin resistance gene. pSR28 (Roels et al. 1992) was digested with Tth111I and AccI, treated with Klenow fragment of DNA polymerase I, and ligated with a 1.3-kb SmaI-Smal fragment from pBEST501 (Itaya et al. 1989 ) harboring a neomycin resistance gene. The resulting plasmid was linearized and then used to transform strain PY79 to neomycin resistance. We confirmed that the resulting strain, SR263, was the product of integration by marker replacement and not single-site reciprocal (Campbell-like) recombination by screening for sensitivity to chloramphenicol, indicating that the chloramphenicol resistance gene in the backbone of pSR28 was not present in the strain.

Strain AD408 (cotEA::cat amyE::cotEepi1), in which the chromosomal copy of $\cot E$ has been replaced with the epitopetagged version of $\cot E$, was created by transforming competent AD28 with linearized pAD105 (see below), selecting for kanamycin resistance, and screening for a disruption of the $a m y E$ gene (Cutting and Vander Horn 1990).

To create strains bearing only the epitope-tagged version of $\cot E$ in combination with one or more sporulation mutations, either chromosomal DNA was prepared from strains bearing the appropriate mutations and used to transform competent strain AD408 to drug resistance or (for the cases of strains AD316, AD407, AD410, and SR287) DNA from strain AD408 was used to transform the appropriate competent sporulation mutant strain to chloramphenicol and kanamycin resistance. To construct SR299, chromosomal DNA from RL244 was used to transform SR287 (see below) to MLS resistance (see below). These constructions resulted in the following strains: AD22 (spoIIAC561::spoIIACRerm cotEA::cat amyE::cotEepi1); AD24 (spoIID::Tn917RHU298 cotEA::cat amyE::cotEepi1); AD25 (spoVID::kn cotEA::cat amyE::cotEepi1); AD27 (spoIIID SermG spoVID::kn cotEA::cat, amyE::cotEepi1); AD316 (spoIIIGA1 cotEA::cat amyE::cotEepi1); AD395 (spoVID::kn amyE::pDG131; see below); AD407 (spoIIBA::erm spoVG::

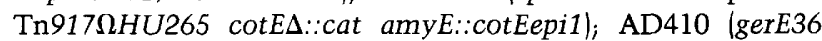
cotEA::cat amyE::cotEepi1); AD466 (spoIIIA::Tn917RHU25

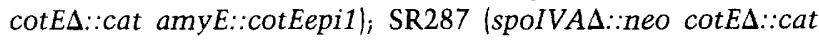

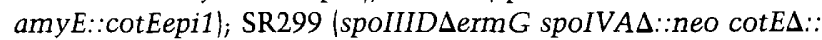
cat amyE::cotEepi1).

Two spoVID null mutant strains, PY79 $2 p S C S$ and spoVID:: $k n$, were built. To create $P Y 79 \Omega p S C S$, a plasmid was first constructed that could be used to generate an internal disruption in spoVID after single reciprocal (Campbell-like) recombination. A 750-bp ClaI-SauIIIA fragment of the spoVID gene (Beall et al. 1993) was subcloned into the plasmid pUS19 (from W. Haldenwang) to create pSCS. To make pUS19, pUC19 was digested with NarI and NdeI and then ligated with a $1.1-\mathrm{kb}$ ClaI-NdeI fragment bearing a spectinomycin resistance gene. The resulting plasmid was used to transform the wild-type strain 168 to spectinomycin resistance. Chromosomal DNA from this strain was used to transform wild-type strain PY79 to spectinomycin resistance. To create spoVID::kn, we first built a plasmid in which part of the spoVID sequence was replaced with a kanamycin resistance gene. pRS32 (Beall et al. 1993) was digested with the restriction enzymes ClaI and AvrII, excising $1.5 \mathrm{~kb}$ of the spoVID open reading frame. pKD102 [from W. Haldenwang; a pBR322 derivative bearing a kanamycin resistance gene from pJH1 (Trieu-Cuot and Courvalin 1983)] was digested with the enzymes SmaI and Sall, liberating a fragment harboring the kanamycin resistance gene. Klenow fragment of DNA polymerase I was used to create blunt ends, and the fragment was ligated into ClaI-AvrII-digested pRS32, also treated with Klenow fragment of DNA polymerase I to create blunt ends. The resulting plasmid was linearized and used to transform PY79 to kanamycin resistance.

\section{General methods}

Recombinant DNA methods were carried out as described by Sambrook et al. (1989). Methods for preparing competent cells, for transformation, and for the preparation of chromosomal DNA in B. subtilis are described by Cutting and Vander Horn (1990). Concentrations of antibiotics for drug selections in $B$. subtilis were at the concentrations recommended by Cutting and Vander Horn (1990). Spectinomycin was used at $50 \mu \mathrm{g} / \mathrm{ml}$.

\section{Insertion of epitope sequence into cotE}

PCR was used to insert the sequence of the hemagglutinin epitope HAl from influenza virus (Field et al. 1988) into the $\cot E$ gene between codons 178 and 179. pADl01 was derived from pGEM3Z (Promega) by digestion with AatII, treatment with T4 DNA polymerase to create blunt ends, and treatment with T4 DNA ligase to create pAD101. An EcoRI-SspI fragment bearing the $\cot E$ gene (Zheng et al. 1988) was then subcloned into EcoRI-SmaI-digested pAD101 to create pAD103, which was used as the template in a PCR. The following primers were synthesized: OLAD1, 5'-AAAAAGACGTCGTAGGGGTAATCTCCCACTAAAAACTCCGG-3' and OLAD2, 5'-AAAAAGA- 
CGTCCCCGACTACGCCAGCCCTGAAGAATAAAAAAGGGACTAGG-3'.

The primers were then purified on a G-50 Sephadex Nick column (Pharmacia) and subjected to PCR under the following conditions: $5 \mu$ lof $10 \times$ buffer $[500 \mathrm{~mm} \mathrm{KCl}, 100 \mathrm{~mm}$ Tris- $\mathrm{HCl}$ (pH 8.3), $15 \mathrm{mM} \mathrm{MgCl}_{2}, 0.01 \%$ (wt/vol) gelatin]; $10 \mu \mathrm{l}$ of dNTP stock containing $0.1 \mathrm{mM}$ each $\mathrm{dNTP} ; 1 \mu \mathrm{l}$ of pADl03 (10 ng); 3.4 $\mu \mathrm{l}$ of OLAD1 $(14.7 \mu \mathrm{M}) ; 5.7 \mu \mathrm{l}$ of OLAD2 $(8.76 \mu \mathrm{M}) ; 24.9 \mu \mathrm{l}$ of $\mathrm{H}_{2} \mathrm{O}$. Two cycles were carried out with annealing at $45^{\circ} \mathrm{C}$, followed by 30 cycles with annealing at $60^{\circ} \mathrm{C}$. Elongation was carried out for $15^{\prime}$. This reaction produced a single product, which was digested with an excess of AatII, treated with T4 DNA ligase, and then transformed into E. coli. The EcoRI-BamHI fragment bearing the insertion mutagenized $\cot E$ gene was then subcloned into pER82 to create pAD105. pER82 is a derivative of pDG364 (Cutting and Vander Horn 1990) bearing a kanamycin resistance gene from pJH1 (Trieu-Cuot and Courvalin 1983) between the EcoRI and SphI sites, designed to allow integration of the subcloned insert by double recombination (marker replacement) into the $B$. subtilis chromosome at the amy $E$ locus (Cutting and Vander Horn 1990).

\section{Insertion of the epitope sequence into spoVID}

An epitope-tagged version of SpoVID was built by first using site-directed mutagenesis (Kunkel 1985) to change the spoVID stop codon from TAA to TTT (at the same time creating a unique DraI site) using the primer 5'-AAAAATAGCCATGCGTTTAAACCGTAAGTGAT-3'. The resulting plasmid was digested with the restriction enzymes $S p h I$ and DraI to liberate a $2.6-\mathrm{kb}$ fragment. pKAepi, containing the sequence of the HAl epitope from hemagglutinin (Field et al. 1988) directly followed by a kanamycin resistance gene, was digested with restriction enzymes $S p h \mathrm{I}$ and $S c a \mathrm{I}$ and ligated with the $2.6 \mathrm{-kb}$ spoVIDbearing fragment to create an in-frame fusion between spoVID and the epitope sequence. The resulting construct was digested with the enzymes SphI and Sall, yielding a $4.1-\mathrm{kb}$ fragment, that was ligated with the amyE-replacement vector pDG364 (Cutting and Vander Horn 1990) that had been digested with restriction enzymes SphI and SalI, to create pDG131. pDG131 was linearized and used to transform strain spoVID::kn to chloramphenicol resistance and loss of amylase production (Cutting and Vander Horn 1990).

\section{Antisera}

The monoclonal antibody $12 \mathrm{CA} 5$ recognizes the HAl epitope of hemagglutinin (Field et al. 1988) and is the kind gift of T. Kirchhausen (Harvard Medical School, Boston, MA). To prepare immunogen for raising anti-SpoIVA antibodies, the spoIVA open reading frame was subcloned downstream of the phage $\mathrm{T} 7$ promoter in the overexpression plasmid pGEMEX-1 (Promega). The plasmid was introduced into strain BL21(DE3)/pLysS (Novagen), and expression of spoIVA was induced as per manufacturers' instructions. Lysates of induced cells were electrophoresed on $8 \%$ polyacrylamide gels. The SpoIVA-containing band was excised, and protein was isolated from the gel slice by electroelution. The eluted protein was then mixed with Freund's adjuvant and injected into the popitial gland of a rabbit according to a standard immunization protocol.

\section{Electron microscopy}

$B$. subtilis was sporulated by exhaustion in Difco sporulation medium (Sandman et al. 1988) for all experiments. Spores were prepared for electron microscopy as described by Margolis et al. (1993).

\section{Immunoelectron microscopy}

Fixation was carried out as described by Driks and Losick (1991) on cells harvested between hours 4 and 5 of sporulation in the case of experiments to localize SpoVID and between hours 7 and 9 of sporulation in all other cases. Dehydration was performed by a modification of the progressive lowering of temperature method of Kellenberger (Hobot et al. 1985), as described in Cutting et al. (1991a), except that infiltration with $100 \%$ Lowicryl K4M and UV irradiation were carried out for 2 days each. Treatment of sectioned cells with antibodies was as described (Cutting et al. 199la), with 5-nm gold-conjugated goat anti-mouse antibodies (Ted Pella) used as a secondary antibody for detection of the 12CA5 monoclonal antibody. The monoclonal antibody 12CA5 was used at a dilution of 1:250 into dilution buffer (Francesconi et al. 1988). Gold grains were enhanced in size using the ENhance kit (Amersham) and a development time of $10^{\prime}$. Cells were stained with freshly made Reynolds lead. As negative controls, wild-type $B$. subtilis (PY79) and mutant strains (AD16, RL325, PM84, PM740, RL64, RL68, RL244, SC500, SR263) fixed at the same time in sporulation as experimental samples were subject to immunoelectron microscopy. In no case was any specific decoration observed. About 0-7 gold grains per cell were present. The anti-SpoIVA antibodies were used at a 1:2500 dilution. As negative controls, this serum was used in immunoelectron microscopy experiments with sporangia bearing a spoIVA mutation (SR263), and a preimmune serum was applied to sporangia that produced SpoIVA (RL244). In both cases, $\sim 0-5$ gold grains per cell were observed.

Immunoelectron microscopy results /with the exception of those concerning SpoVID/ are representative of experiments in which $50-80 \%$ of the cells showed significant decoration. In experiments to localize SpoVID, only $\sim 0.5 \%$ of the cells showed significant decoration.

\section{Acknowledgments}

We thank Richard Gardner for advice about PCR; Thomas Kirchhausen, William Haldenwang, and Leslie Berg for gifts of reagents; Jean Greenberg and Jeanine Maddock for helpful discussions; and Patrick Piggot for comments on the manuscript. This work was supported by National Institutes of Health (NIH) grants GM18568 to R.L. and AI20319 to C.P.M. and by an NIH postdoctoral fellowship to B.B.

The publication costs of this article were defrayed in part by payment of page charges. This article must therefore be hereby marked "advertisement" in accordance with 18 USC section 1734 solely to indicate this fact.

\section{References}

Alley, M.R.K., J.R. Maddock, and L. Shapiro. 1993. Requirement of the carboxyl terminus of a bacterial chemoreceptor for its targeted proteolysis. Science 259: 1754-1757.

Aronson, A.I. and P. Fitz-James. 1976. Structure and morphogenesis of the bacterial spore coat. Bacteriol. Rev. 40: 360402.

Beall, B., A. Driks, R. Losick, and C.P. Moran Jr. 1993. Cloning and characterization of a gene required for assembly of the Bacillus subtilis spore coat. J. Bacteriol. 175: 1705-1716.

Buchanan, C.E. and A. Gustafason. 1992. Mutagenesis and mapping of the gene for a sporulation-specific penicillin-binding 
protein in Bacillus subtilis. J. Bacteriol. 174: 5430-5435.

Chant, J. and J.R. Pringle. 1991. Budding and cell polarity in Saccharomyces cerevisiae. Curr. Opin. Gen. Dev. 1: 342350.

Coote, J.G. 1972. Sporulation in Bacillus subtilis. Characterization of oligosporogenous mutants and comparison of their phenotypes with those of asporogenous mutants. J. Gen. Microbiol. 71: 1-15.

Cutting, S. and P.B. Vander Horn. 1990. Molecular biological methods for Bacillus. John Wiley, Chichester, UK.

Cutting, S., S. Panzer, and R. Losick. 1989. Regulatory studies on the promoter for a gene governing synthesis and assembly of the spore coat in Bacillus subtilis. J. Mol. Biol. 207: 393404.

Cutting, S., A. Driks, R. Schmidt, B. Kunkel, and R. Losick. 1991 a. Forespore-specific transcription of a gene in the signal transduction pathway that governs pro- $\sigma^{\mathrm{K}}$ processing in $\mathrm{Ba}$ cillus subtilis. Genes \& Dev. 5: 456-466.

Cutting, S., L. Zheng, and R. Losick. 1991b. Gene encoding two alkali-soluble components of the spore coat Bacillus subtilis. J. Bacteriol. 173: 2915-2919.

Driks, A. and R. Losick. 1991. Compartmentalized expression of a gene under the control of sporulation transcription factor ${ }^{\mathrm{E}}$ in Bacillus subtilis. Proc. Natl. Acad. Sci. 88: 9934 9938.

Field, J., J.-I. Nikawa, D. Broek, B. MacDonald, L. Rogers, I.A. Wilson, R.A. Lerner, and M. Wigler. 1988. Purification of a RAS-responsive adenylyl cyclase complex from Saccharomyces cerevisiae by use of an epitope addition method. Mol. Cell. Biol. 8: 2159-2165.

Francesconi, S.C., T.J. MacAlister, B. Setlow, and P. Setlow. 1988. Immunoelectron microscopic localization of small, acid-soluble spore proteins in sporulating cells of Bacillus subtilis. J. Bacteriol. 170: 5963-5967.

Hobot, J.A., W. Villiger, J. Escaig, M. Maeder, A. Ryter, and E. Kellenberger. 1985. Shape and structure of nucleoids observed on sections of ultrarapidly frozen and cryosubstituted bacteria. J. Bacteriol. 162: 960-971.

Illing, N. and J. Errington. 1991. Genetic regulation of morphogenesis in Bacillus subtilis: Roles of $\sigma^{\mathrm{E}}$ and $\sigma^{\mathrm{F}}$ in prespore engulfment. /. Bacteriol. 173: 3159-3169.

Itaya, M., K. Kondo, and T. Tanaka. 1989. A neomycin resistance gene cassette selectable in a single copy state in the Bacillus subtilis chromosome. Nucleic Acids Res. 17: 4410.

Kroos, L., B. Kunkel, and R. Losick. 1989. Switch protein alters specificity of RNA polymerase containing a compartmentspecific sigma factor. Science 243: 526-529.

Kunkel, B., K. Sandman, S. Panzer, P. Youngman, and R. Losick. 1988. The promoter for a sporulation gene in the spoIVC locus of Bacillus subtilis and its use in studies of temporal and spatial control of gene expression. $J$. Bacteriol. 170: 3513-3522.

Kunkel, B., L. Kroos, H. Poth, P. Youngman, and R. Losick. 1989. Temporal and spatial control of the mother-cell regulatory gene spoIIID of Bacillus subtilis. Genes \& Dev. 3: 1735-1744.

Kunkel, T.A. 1985. Rapid and efficient site-specific mutagenesis without phenotypic selection. Proc. Natl. Acad. Sci. 82: 488-492.

Losick, R. and P. Stragier. 1992. Crisscross regulation of celltype-specific gene expression during development in Bacillus subtilis. Nature 355: 601-604.

Losick, R., P. Youngman, and P.J. Piggot. 1986. Genetics of endospore formation in Bacillus subtilis. Annu. Rev. Genet. 20: 625-669.

Lutkenhaus, J. 1993. FtsZ ring in bacterial cytokinesis. Mol.
Microbiol. 9: 403-410.

Margolis, P., A. Driks, and R. Losick. 1991. Establishment of cell type by compartmentalized activation of a transcription factor. Science 254: 562-565.

1993. Sporulation gene spollB from Bacillus subtilis. $J$. Bacteriol. 175: 528-540.

Moir, A. 1981. Germination properties of a spore coat-defective mutant of Bacillus subtilis. I. Bacteriol. 146: 1106-1116.

Piggot, P.J. and J.G. Coote. 1976. Genetic aspects of bacterial endospore formation. Bacteriol. Rev. 40: 908-962.

Roels, S., A. Driks, and R. Losick. 1992. Characterization of spoIVA, a sporulation gene involved in coat morphogenesis in Bacillus subtilis. J. Bacteriol. 174: 575-585.

Sambrook, J., E.F. Fritsch, and T. Maniatis. 1989. Molecular cloning: A laboratory manual. Cold Spring Harbor Laboratory Press, Cold Spring Harbor, New York.

Sandman, K., P. Youngman, and R. Losick. 1987. Genetic analysis of Bacillus subtilis spo mutations generated by Tn917mediated insertional mutagenesis. Genetics 117: 603-617.

Sandman, K., L. Kroos, S. Cutting, P. Youngman, and R. Losick. 1988. Identification of the promoter for a spore coat protein gene in Bacillus subtilis and studies on the regulation of its induction at a late stage of sporulation. I. Mol. Biol. 200: $461-473$.

Shapiro, L. 1993. Protein localization and asymmetry in the bacterial cell. Cell 73: 841-856.

Stevens, C.M., R. Daniel, N. Illing, and J. Errington. 1992. Characterization of a sporulation gene, spoIVA, involved in spore coat morphogenesis in Bacillus subtilis. I. Bacteriol. 174: 586-594.

Trieu-Cuot, P. and P. Courvalin. 1983. Nucleotide sequence of the Streptococcus faecalis plasmid gene encoding the $3^{\prime} 5^{\prime \prime}$ aminoglycoside transferase type III. Gene 23: 331-341.

Youngman, P., J.B. Perkins, and R. Losick. 1984. A novel method for the rapid cloning in Escherichia coli of Bacillus subtilis chromosomal DNA adjacent to Tn917 insertions. Mol. \& Gen. Genet. 195: 424-433.

Zheng, L. and R. Losick. 1990. Cascade regulation of spore coat gene expression in Bacillus subtilis. J. Mol. Biol. 212: 645660.

Zheng, L., W.P. Donovan, P.C. Fitz-James, and R. Losick. 1988. Gene encoding a morphogenic protein required in the assem bly of the outer coat of the Bacillus subtilis endospore. Genes \& Dev. 2: 1047-1054.

Zheng, L., R. Halberg, S. Roels, H. Ichikawa, L. Kroos, and R. Losick. 1992. Sporulation regulatory protein GerE from $\mathrm{Ba}$ cillus subtilis binds to and can activate or repress transcription from promoters for mother-cell-specific genes. $J$. Mol. Biol. 226: 1037-1050. 


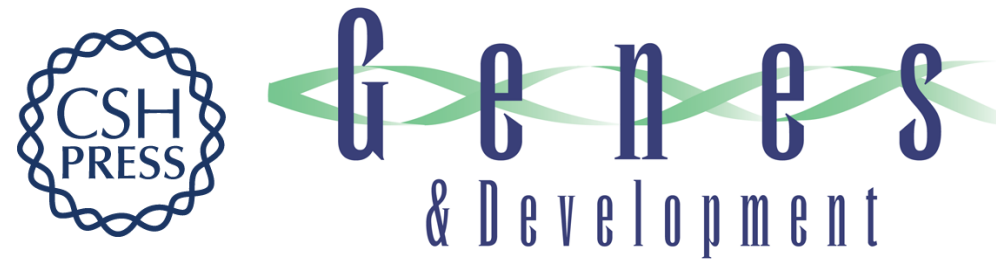

\section{Subcellular localization of proteins involved in the assembly of the spore coat of Bacillus subtilis.}

A Driks, S Roels, B Beall, et al.

Genes Dev. 1994, 8:

Access the most recent version at doi:10.1101/gad.8.2.234

References This article cites 36 articles, 23 of which can be accessed free at:

http://genesdev.cshlp.org/content/8/2/234.full.html\#ref-list-1

License

Email Alerting

Service

Receive free email alerts when new articles cite this article - sign up in the box at the top right corner of the article or click here.

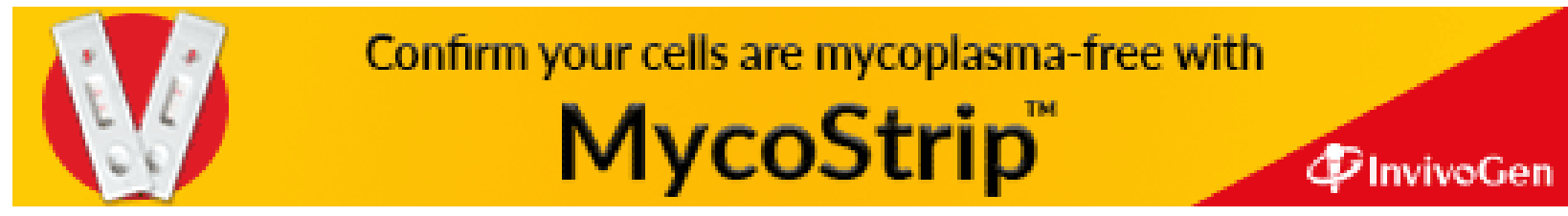

